\title{
Skandinavische Beschäftigungspolitik - Stärken und Schwächen im internationalen Vergleich
}

\begin{abstract}
Die skandinavischen Länder dienen erneut als Vorbilder für eine erfolgreiche Beschäftigungs- und Arbeitsmarktpolitik in Europa. Sowohl ihre wirtschaftliche Leistungsfähigkeit wie auch ihre wohlfahrts- und beschäftigungspolitische Innovationskraft ziehen das Augenmerk auf sich. Worin besteht das nordische „Beschäftigungswunder“ im Detail? Welche Maßnahmen und Instrumente haben im Norden Europas erneut weitgehend Vollbeschäftigung ermöglicht, obwohl auch diese Länder von den ökonomischen Krisen der frühen 1990er Jahre und dem damit einhergehenden Beschäftigungsabbau betroffen waren? Wo liegen die Stärken, wo die Schwächen des nordischen Reformweges? Und schließlich, was kann Deutschland beschäftigungspolitisch vom Norden lernen? Diese Fragen stehen im Zentrum des folgenden Beitrages.
\end{abstract}

\section{Einleitung}

Bereits in den 1980er Jahren dienten einige Länder des Nordens als beschäftigungspolitische Reformvorbilder. Einzig Dänemark galt damals als europäisches „Sorgenkind“, da dort Massenarbeitslosigkeit, hohe Inflationsraten sowie beachtliche Defizite der öffentlichen Haushalte vorherrschten. Mit den ökonomischen Krisen der frühen 1990er Jahre stieg jedoch auch in den anderen nordischen Ländern die Arbeitslosigkeit deutlich an (Jochem 1998). Im Jahr 1994, am Höhepunkt der damaligen Beschäftigungskrise, lag die offene Arbeitslosigkeit in Dänemark bei $12 \%$, in Schweden bei $8 \%$ und in Finnland gar bei $18 \%$. Einzig in Norwegen und Island schien sich die offene Arbeitslosigkeit in Grenzen zu halten. Hier stiegen die entsprechenden Werte nicht wesentlich über die $5 \%$-Marke. Dennoch, der Norden verlor im Zusammenhang mit der ökonomischen Krise der frühen 1990er Jahre an reformpolitischer Anziehungskraft. Es schien sich anzudeuten, dass der nordische Sonderweg an sein Ende gelangt wäre - so wie es viele Kritiker prophezeit hatten.

Wie Phönix aus der Asche stellen sich die nordischen Länder ein Jahrzehnt später in internationalen Vergleichen und Benchmarking-Berichten dar (vgl. u.a. Palme 1999; Becker 2008; Becker/Schwartz 2005; Merkel et al. 2006). Im Norden folgte auf die Krise der 1990er Jahre ein dynamisches Wirtschaftswachstum. In kurzer Zeit konnten die öffentlichen Haushalte erfolgreich konsolidiert werden. Die Wohlfahrtsstaaten wurden reformiert, ihr genuin sozialdemokratisch-egalitärer Charakter blieb allerdings weitgehend erhalten. Auch wenn diesbezüglich die Expertenmeinungen geteilt sind, so ist offensichtlich, dass Ungleichheit und ökonomische Armut nach wie vor weitgehend sozialpolitisch eingedämmt sind, vor allem wenn man die einschlägigen Kennzahlen des Nordens mit denen der kontinentaleuropäischen Länder vergleicht.

Die Arbeitsmärkte erholten sich rasch, und niedrige Arbeitslosenraten gingen in weiten Teilen des Nordens erneut einher mit einer sehr hohen Integration der Bevölkerung im erwerbsfähigen Alten (d.h. im Alter von 15 bis 64 Jahren) in den Arbeitsmarkt. Diese hohe Arbeitsmarktintegration zeichnet sich zudem durch eine hohe Gleichheit zwischen den Geschlechtern aus. Und Dänemark, das in der Nachkriegszeit stets im Schatten des schwedischen Modells stand, steht heute für ein viel bewundertes arbeitsmarktpolitisches Vorzeigemodell in Europa, das in der Literatur als "Flexicurity“ benannt und diskutiert wird (wenngleich der Begriff ursprünglich aus der niederländischen Reformdebatte der 1990er Jahre stammt).

Im Folgenden werden die einzelnen Bausteine dieser nordischen Erfolgsgeschichten analysiert. Worin lagen die Ursachen für die ökonomische Erholung? Was steuerten Politik und Sozialpartner hierzu bei? Und wo liegen die Stärken, wo die Schwächen des nordischen Erfolgsmodells? Gleich zu Beginn muss jedoch mit Nachdruck betont werden, dass diese Analyse eines „nordischen Modells“ nicht die Unter- schiede zwischen den nordischen Staaten und Regionen verwischen will. Der Norden war und ist heute mehr denn je ein heterogener politischer und ökonomischer Raum. Finnland und Schweden zum Beispiel unterscheiden sich in vielen wichtigen ökonomischen und politischen Aspekten sehr deutlich voneinander. Und der Erdölreichtum eröffnet der norwegischen Politik große Gestaltungsspielräume und Freiheiten, wie sie sonst weder im Norden noch in Kontinentaleuropa vorzufinden sind.

Trotz dieser intra-nordischen Unterschiede lassen sich dennoch weitgehende nordische Gemeinsamkeiten ausmachen, wenn die skandinavischen Staaten mit anderen OECD-Staaten verglichen werden. Dies soll in diesem Beitrag anhand beschäftigungspolitisch relevanter Politikfelder und empirischer Kennzahlen geschehen. In einem ersten Schritt werden die beschäftigungspolitischen Charakteristika der nordischen Länder aus einer vergleichenden Perspektive erörtert (Abschnitt 2). Daran anschließend erfolgt eine Analyse der nordischen Reformwege im Bereich der Wirtschafts-, Sozial- sowie Lohnpolitik (Abschnitt 3). Der vierte Abschnitt ist der Arbeitsmarktpolitik sowie insbesondere der dänischen Flexicurity gewidmet. Im

\footnotetext{
Sven Jochem, PD, Dr., vertritt eine Professur für Policy-Analyse und Politische Theorie am Fachbereich Politik- und Verwaltungswissenschaften der Universität Konstanz. Arbeitsschwerpunkte: Vergleichende Analyse wohlfahrtsstaatlicher Politik, normative und empirische Demokratietheorien. e-mail: sven.jochem@uni-konstanz.de
} 
Tabelle 2: Arbeitsmarktprofile des Nordens - in \% -

\begin{tabular}{|c|c|c|c|c|c|c|}
\hline & \multirow{2}{*}{$\begin{array}{c}\text { BQ } \\
55-64 \\
2007\end{array}$} & \multirow{2}{*}{$\begin{array}{l}\text { AAMP } \\
2007\end{array}$} & \multicolumn{4}{|c|}{ Reales durchschnittliches Lohnwachstum } \\
\hline & & & 1990-1995 & 1995-2000 & 2000-2005 & 2006 \\
\hline Australien & 56,7 & 0,34 & 1,0 & 1,9 & 1,6 & 2,3 \\
\hline Österreich & 38,6 & 0,71 & 1,8 & 0,4 & 0,9 & 1,9 \\
\hline Belgien & 33,8 & 1,09 & 1,8 & 1,3 & 0,3 & 1,3 \\
\hline Kanada & 57,1 & 0,31 & $-0,2$ & 2,0 & 1,2 & 2,5 \\
\hline Dänemark & 58,7 & $1,85^{1)}$ & 0,8 & 1,6 & 1,5 & 1,9 \\
\hline Finnland & 55,0 & 0,89 & 0,0 & 1,3 & 2,5 & 1,8 \\
\hline Frankreich & 37,9 & 0,92 & 1,1 & 1,3 & 1,3 & 0,9 \\
\hline Deutschland & 52,0 & 0,88 & 2,1 & 0,8 & 0,2 & $-0,3$ \\
\hline Griechenland & 42,1 & - & 3,3 & 1,9 & 3,4 & 3,5 \\
\hline Island & 84,9 & - & - & - & - & - \\
\hline Irland & 54,1 & 0,61 & 2,5 & 1,9 & 2,9 & 1,6 \\
\hline Italien & 33,8 & 0,53 & $-0,7$ & 0,8 & 0,2 & $-0,2$ \\
\hline Japan & 66,1 & 0,19 & 1,3 & 0,5 & 0,2 & 0,1 \\
\hline Luxemburg & 34,3 & 0,49 & 1,9 & 1,2 & 1,5 & 0,8 \\
\hline Niederlande & 50,1 & 1,22 & 0,3 & 0,0 & 0,5 & $-0,1$ \\
\hline Neuseeland & 72,0 & 0,38 & - & - & - & - \\
\hline Norwegen & 69,0 & 0,58 & 1,2 & 1,9 & 3,6 & 2,3 \\
\hline Portugal & 50,9 & 0,61 & 1,6 & 2,6 & 0,4 & $-2,6$ \\
\hline Spanien & 44,6 & 0,80 & 1,9 & $-0,5$ & $-0,4$ & $-0,7$ \\
\hline Schweden & 70,1 & 1,36 & $-0,2$ & 3,2 & 1,5 & 2,1 \\
\hline Schweiz & 67,2 & 0,67 & 0,9 & 1,0 & 1,2 & 1,4 \\
\hline UK & 57,4 & 0,42 & 0,9 & 2,5 & 1,6 & 1,7 \\
\hline USA & 61,8 & 0,14 & 1,0 & 2,9 & 0,2 & 1,7 \\
\hline \multicolumn{7}{|c|}{$\begin{array}{l}\text { Anmerkung: BQ 55-64 = Beschäftigungsquote der älteren Arbeitnehmer von } 55 \text { bis } 64 \text { Jahren in \% der altersspezifischen } \\
\text { Population; AAMP = Ausgaben für aktive Arbeitsmarktpolitik in \% des Bruttoinlandsproduktes. - Wert liegt nicht vor. }\end{array}$} \\
\hline
\end{tabular}

aber stehen diese Zahlen in einem deutlichen Kontrast zur kontinentaleuropäischen Massenarbeitslosigkeit, wie sie in Frankreich sowie in (Ost-) Deutschland zu beobachten ist.

Als weiterer Beleg für die gute nordische Arbeitsmarktdynamik gilt, dass sich dort das Problem der Langzeitarbeitslosigkeit nicht so belastend auswirkt wie zum Beispiel in Deutschland, Italien oder Frankreich. Dies mag viele Gründe haben, allerdings scheinen die aktive Arbeitsmarktpolitik bzw. die Flexicurity sowie die Bildungspolitik im Norden wichtige Ursachen für die dortige geringe Langzeitarbeitslosigkeit zu sein (Abschnitt 3). Es ist beeindruckend, dass in allen nordischen Ländern nach 1994 der Anteil der Langzeitarbeitslosen deutlich reduziert werden konnte, während der Trend in Deutschland eher für eine Ausweitung dieses Arbeitsmarktproblems spricht (Tabelle 2). Es kann hier nicht im Detail auf die viel diskutierten Folgen der Langzeitarbeitslosigkeit eingegangen werden. $\mathrm{Zu}$ Recht werden sie aber in der bundesdeutschen Reformdebatte als Standortnachteil mit hohen sozialen sowie ökonomischen Kosten - für die Gesellschaft und die betroffenen Personen - diskutiert und kritisiert. Hier scheint die nordische Politik tatsächlich eine Arbeits- marktdynamik zu ermöglichen, die nicht dem angelsächsischen Weg folgt, der auf großen Lohndifferenzen und einer rudimentären sozialen Sicherung basiert. Im Gegenteil wird diese Dynamik im Norden ermöglicht bei relativ hoher Lohngleichheit sowie einer weitgehend umfassenden sozialen Absicherung der Arbeitslosen.

\subsection{INVESTITIONEN IN AUS- UND WEITERBILDUNG}

Charakteristisch für die nordischen Länder ist ferner die große Bedeutung aktiver Arbeitsmarktpolitik. Die nordischen Staaten - erneut unabhängig von der politischen Regierungsfärbung - setzten und setzen mit unterschiedlicher Gewichtung auf eine aktive Arbeitsmarktpolitik mit starker Betonung der Aus- und Weiterbildungsmaßnahmen. Dass dies keine symbolische Politik ist, wird deutlich, wenn die relativen Ausgaben für eine solche Politik mit anderen Ländern verglichen werden (Tabelle 2). Trotz deutlich größerer Arbeitsmarktprobleme liegen die bundesdeutschen Ausgaben (gemessen als Anteil am Bruttoinlandsprodukt) hierfür wesentlich unter dem dänischen und schwedischen Niveau. Die finnischen Aufwendungen ähneln dem deutschen Niveau, und Norwegen rangiert im

unteren Mittelfeld der Verteilung. Arbeitsmarktdynamik unter den Bedingungen einer soliden sozialen Absicherung, ohne verbreitetes Armutsrisiko und soziale Verwerfungen, ist nicht zum budgetären Nulltarif zu erlangen.

\section{Wirtschaftliche Leistungspotenziale}

Aus vergleichender Perspektive kann die These vertreten werden, dass im Norden die Arbeitsmarktintegration beider Geschlechter unter den Bedingungen starker Lohngleichheit und umfangreicher sozialer Sicherung institutionell sowie kulturell abgesichert ist. Worauf diese beeindruckende Performanz innerhalb der ökonomischen Rahmenbedingungen in Europa zurückgeführt werden kann, ist Thema des folgenden Abschnittes.

\subsection{STABILITÄTSORIENTIERTE WIRTSCHAFTSPOLITIK}

Einstmals stand der Norden abseits des wirtschaftspolitischen Mainstreams in Europa. Die zentralen Freiräume der nordischen Wirtschaftspolitik im Zeitalter noch weitgehend intakter Ländergrenzen lagen in einer autonomen Währungs-, Geld- sowie Zinspolitik, welche nach politischen Zielen der Regierungen und nicht nach unabhängig-ökonomischen Urteilen der Zentralbanken ausgestaltet wurden. Mit der fortschreitenden europäischen Integration und den durchlässiger gewordenen Grenzen für Gesellschaft, Ökonomie und Politik sind diese nordischen Sonderwege unbegehbar geworden.

In den 1980er Jahren erfolgten bedeutsame wirtschaftspolitische Kursänderungen im Norden. Dezidiert und zielstrebig unter den bürgerlichen Regierungen in Dänemark, kurzfristig und zum Teil von der Macht des Faktischen getrieben in Schweden und Finnland. Mit der Mitgliedschaft in der Europäischen Union (EU) der beiden zuletzt genannten Länder gelten nun für alle nordischen Regierungen die Imperative einer europäischen stabilitätsorientierten Wirtschafts-, Währungs- und Zinspolitik. Dies gilt weitgehend auch für die Nichtmitglieder im Norden, Norwegen und Island. Beide Länder können kaum große Abweichungen vom europäischen 
Mainstream riskieren. $\mathrm{Zu}$ groß wären die Risiken einer spekulativen Abstrafung unter den Bedingungen offener und volatiler Kapitalmärkte.

Eine stabilitätsorientierte Wirtschaftspolitik wird heute im Norden weitgehend mit Bravour umgesetzt. Unter anderem lässt sich dies auch an den dortigen Konsolidierungserfolgen der öffentlichen Haushalte ablesen. Insbesondere die finnischen Regierungen verfolgten diese Konsolidierungspolitik trotz tiefer ökonomischer Krise mit Nachdruck - flankiert von einer korporatistischen Sozialpartnerschaft. Und im Gegensatz zu den nordischen Nachbarländern ist es vor allem die finnische Politik, die sich in der europäischen Wirtschaftsund Währungsunion als „Musterknabe“ darzustellen versucht - Finnland ist das einzige nordische Land im Euroraum.

\subsection{LOHNPOLITIK}

Auch die ehemals nationalstaatlich fokussierte Lohnpolitik im Norden hat sich zusehends europäisiert. Noch in den 1980er Jahren wurden lohnpolitische Sonderwege im Norden beschritten - oft mit der Hoffnung, mögliche negative Folgen für die Konkurrenzfähigkeit der exportorientierten Industrie durch strategische Abwertungen der nationalen Währungen korrigieren zu können. Durch den Entzug dieses strategischen Korrektivs steht die nordische Lohnpolitik vor der Aufgabe, sowohl die nationalen Verteilungsspielräume zu beobachten als auch die Auswirkungen auf die Konkurrenzkraft der Ökonomie nicht aus den Augen zu verlieren. Erneut waren es die dänischen Regierungen bürgerlicher Prägung in den 1980er Jahren, die mit politischem Druck die Sozialpartner dazu bewegten, die internationale Perspektive verstärkt zu berücksichtigen. Nach niederländischem Vorbild wurden dort die ersten Standortpakte in Europa geschlossen, die neben sozialpolitischen Reformprojekten dezidiert eine Lohnmäßigung und Ankoppelung der dänischen Lohnentwicklung an die Vorgaben der Haupthandelsländer verfolgten (Siegel 2005; Hassel 2006; Jochem i. E.).

Eine korporatistische und stark zentralisierte Lohnpolitik war lange Zeit ein hervorstechendes Charakteristikum des Nordens. Zumindest werden die skandinavischen Länder von verschiedenen Experten immer der Spitzengruppe des internationalen Korporatismus zugeordnet (Siaroff 1999; Kenworthy 2000). Der nordische
Korporatismus hat sich jedoch in den vergangenen Dekaden deutlich verändert (vgl. den Beitrag von Mailand in diesem Heft). Die sogenannten neuen sozialen Pakte, die als eine Art Renaissance tripartistischer Konzertierung unter den veränderten ökonomischen und politischen Rahmenbedingungen in Europa angesehen werden können, sind einzig in Dänemark während der 1990er Jahre sowie in Finnland bis $2007 \mathrm{zu}$ beobachten. Dort konnten Aspekte der Lohnpolitik mit sozial- und arbeitsmarktpolitischen Reformen kombiniert werden. Als ein Effekt kann eine Mäßigung der Lohnsteigerungen in diesen Ländern ausgemacht werden. Eine solche Konzertierung in einem neuen sozialen Pakt ist hingegen in Schweden gescheitert. Allerdings konnte dort die Tradition einer bipartistischen Konzertierung im Industriesektor neu belebt werden, die aufgrund der Lohnführerschaft dieses Sektors Ausstrahlungseffekte auf die übrigen Wirtschaftsteile hat (Jochem 2003).

Insgesamt ist die aktuelle Lohnpolitik im Norden stärker dezentralisiert als in den 1970er und 1980er Jahren (Elvander 2002). Wie in vielen anderen Ländern Europas wurde die Lohnfindung zum Teil individualisiert und die Konkurrenz zwischen den Sektoren nahm deutlich zu. Trotzdem kann die Lohnentwicklung im Norden während der 1990er Jahre kaum als inflationär oder expansiv bezeichnet werden (vgl. die entsprechenden Daten in Tabelle 2). Ganz im Gegenteil haben die (erzwungenen, nicht strategisch angestrebten) Abwertungen der nationalen Währungen in Schweden und Finnland Anfang der 1990er Jahre diese deutlichen Wettbewerbsvorteile durch eine gemäßigte Lohnpolitik konserviert. Nicht zuletzt darauf können die Erfolge beider Länder in der ökonomischen Rekonvaleszenz zurückgeführt werden. $\mathrm{Zu}$ Beginn des 21. Jahrhunderts schwächte sich die Lohnmäßigung im Norden jedoch ab. Die zur Verfügung stehenden Daten zeigen, dass im Norden, vor allem in Norwegen und in Finnland, die Lohnsteigerungen jüngst deutlich über dem OECD-Durchschnitt sowie den jeweiligen Haupthandelsländern lagen (Tabelle 2). Dies kann als ein Indiz dafür interpretiert werden, dass die sich aufweichenden Institutionen des nordischen korporatistischen Lohnfindungsmodells gegenwärtig Lohnentwicklungen zulassen, die mittel- und langfristig die Wettbewerbsfähigkeit der exportorientierten Sektoren unterminieren könnten.
Dennoch, die ökonomische Erholung sowie die rasche Behebung der Beschäftigungskrise in Dänemark und Finnland (sowie Anfang der 1990er Jahre in Schweden) sind auch die Erfolge einer sozialpartnerschaftlich flankierten Politik der relativen Lohnmäßigung.

\subsection{STEUERPOLITIK UND INNOVATIONSKRAFT}

Ein weiteres Charakteristikum der nordischen Wirtschaftspolitik ist die hohe Steuerlast (Ganghof 2006; 2007). Obwohl seit den 1980er Jahren die Spitzensteuersätze bei den Einkommenssteuern sowie insgesamt die Besteuerung der Unternehmen in vielen Ländern des Nordens zum Teil deutlich abgesenkt wurden, ist die volkswirtschaftliche Gesamtsteuerlast immer noch beträchtlich. Dies hat auch damit zu tun, dass die nordischen Wohlfahrtsstaaten zu großen Teilen über Steuern finanziert werden. Es überrascht wenig, dass politischer Protest gegen die hohe Steuerlast im Norden aufgekommen ist. Insbesondere die rechtspopulistischen Parteien in Norwegen und Dänemark können dieses Mobilisierungspotenzial für sich nutzen. Gleichwohl ist auch darauf zu verweisen, dass zum Beispiel die schwedischen Konservativen nach ihrem Wahlsieg 2006 nicht der Versuchung rascher Steuersenkungen erlagen (Jochem 2006).

Die hohe Steuerlast ist ein sehr wichtiger Pfeiler des nordischen Modells. Trotzdem ist die wirtschaftliche Performanz des Nordens beeindruckend. Dies ist für weite Teile der ökonomischen Forschung ein schwer zu lösendes Rätsel. Offensichtlich ist die hohe Besteuerung - wenn auch seitens der Firmen vielfach beklagt - kein gravierendes Hemmnis für die wirtschaftliche Innovationskraft des Nordens, wie Studien zu ihrer Innovations- und Wettbewerbsfähigkeit zeigen (vgl. die einschlägigen Beiträge in Becker/Schwartz 2005; Merkel et al. 2006; Becker 2008).

\subsection{MODERNISIERUNGSPFADE}

Letztlich sollte nicht übersehen werden, dass die wirtschaftliche Leistungskraft teilweise auch durch historische Pfadabhängigkeiten mit geprägt ist (Schwartz 2001; Becker 2008; Becker/Schwartz 2005). Ausgehend von der ökonomischen Restrukturierung während der 1960er und 1970er Jahre gelang den nordischen Ländern früh, 
und deutlich eher als vielen Ländern Kontinentaleuropas, ein Wandel hin zu modernen Dienstleistungsökonomien (Iversen/ Wren 1998) - einschließlich expandierender Dienstleistungen im öffentlichen Sektor. Die Dienstleistungsorientierung der nordischen Wohlfahrtsstaaten hat hier ihren historischen Ursprung (Sipilä 1997).

Hieraus erklärt sich auch der hohe Anteil der öffentlichen Beschäftigung an der Gesamtbeschäftigung im Norden. Selbst wenn die statistische Grenzziehung zwischen öffentlichem und privatem Beschäftigungssektor nicht unproblematisch ist (OECD 2008b), so zeigen doch jüngere Studien, dass die genuin öffentliche Beschäftigung im Jahr 2005 in Schweden und Norwegen knapp unter 30 \% der Gesamtbeschäftigung ausmacht. Entsprechende Werte für Deutschland oder Österreich liegen bei ca. $10 \%$ (OECD 2008b, S. 20). Im historischen Vergleich kann zudem festgestellt werden, dass sich diese Werte seit 1995 für die OECD-Länder, für die Daten vorliegen, nur unwesentlich veränderten (OECD 2008b, S. 21). Dieser hohe Anteil der öffentlichen Beschäftigung bewirkt eine kaum zu unterschätzende Dämpfung ökonomischer Krisen. Vor allem die hohe nordische Frauenerwerbstätigkeit geht auf die öffentlichen Dienstleistungen der nordischen Wohlfahrtsstaaten zurück.

Just dieser hohe Anteil staatlicher Beschäftigung und die prominente Rolle des Staates als Arbeitgeber ziehen Kritik auf sich. In Medien und Wissenschaft wird diese Beschäftigung als nicht effizient bewertet. Tatsächlich haben die nordischen (auch sozialdemokratischen) Regierungen der vergangenen Dekaden begonnen, die jeweiligen öffentlichen Sektoren zu reformieren (Klitgaard 2007). Hiermit gehen neue Managementmethoden einher sowie moderate Privatisierungen (Green-Pedersen 2002), wie beispielsweise die des Gesundheitssektors in Schweden seit den 1990er Jahren (Jochem 2003). Damit wird zwar die Dominanz öffentlicher Beschäftigung im Norden (noch) nicht unterminiert. Allerdings lassen sich erste Zeichen ausmachen, dass sich der Staat als Arbeitgeber auch im Norden auf dem vorsichtigen Rückzug befindet.

\subsection{AKTIVE FORSCHUNGS- UND BILDUNGSPOLITIK}

Die aktive Rolle des Staates lässt sich zudem an der gezielten Förderung von Bildung und Forschung im Norden ablesen. Nach Angaben der OECD sind die nordischen Länder stets in der Spitzengruppe vertreten, wenn die Ausgaben für Bildung sowie für Forschung und Entwicklung analysiert werden (OECD 2008c). So liegen die schwedischen und finnischen Ausgaben für Forschung und Entwicklung im Jahr 2005 bei ca. 3,5\% des Bruttoinlandproduktes (BIP). Deutschland kommt hier lediglich auf einen Wert von ca. 2,5\%. Noch deutlicher sind die Unterschiede im Bereich der Bildungspolitik. Werden hier die Ausgaben (in Relation zum Bruttoinlandprodukt) im Jahr 2004 analysiert, dann sind die nordischen Länder - mit dem isländischen Spitzenreiter - in der Spitzengruppe vertreten. Sie geben zu diesem Zweck zwischen $6 \%$ und $8 \%$ des Bruttoinlandsproduktes aus. Anders hingegen die Situation in Deutschland. Hier liegt der Wert für das Referenzjahr lediglich bei knapp über $5 \%$ (alle Daten aus OECD 2008c).

Die Regierungen des Nordens weiteten ihre Bildungssysteme gezielt seit den 1990er Jahren aus. Dies hat als „human investment strategy" zur Folge, dass der Anteil der geringqualifizierten Arbeitnehmer tendenziell geringer ist als in Kontinentaleuropa. Und gerade diese Gruppe hat auf dem Arbeitsmarkt die größten Beschäftigungsprobleme bzw. sieht sich mit der Gefahr konfrontiert, in Langzeitarbeitslosigkeit abzurutschen. Die Bildungspolitik des Nordens flankiert also dynamische Arbeitsmärkte und wirkt tendenziell gegen etwaige Langzeitarbeitslosigkeit. Die staatlichen Aktivitäten im Bereich der Forschung zielen ferner direkt in Richtung Produktinnovation sowie Schaffung hoch qualifizierter Arbeitsplätze. Nicht zuletzt die vor allem in Schweden und Finnland stark vertretene IT-Industrie ist ein Indikator einer solchen Bildungs- und Beschäftigungsstrategie. Bildung und Beschäftigungsdynamik im IT-Sektor haben die klassischen industriellen Beschäftigungsmodelle im Norden abgelöst, man kann gegenwärtig - auf Schweden bezogen - von einem „digitalen Volksheim“ (DER SPIEGEL 19/2000) sprechen.

\subsection{ZWISCHENFAZIT}

Zusammenfassend kann formuliert werden, dass die nordischen Erfolgsgeschichten ökonomischer Performanz und Reformpolitik viele Ursachen haben. Frühere Politikinstrumente allerdings, wie eine au- tonome Zins- und Währungspolitik, stehen nicht mehr zur Verfügung, da die Europäisierung der Wirtschaftspolitik vor den nordischen Ländern nicht halt gemacht hat. Positiv wirken vielmehr die Effekte einer lange Zeit konzertierten und gemäßigten Lohnpolitik, die weiterhin relativ harmonischen Strategien der Sozialpartner sowie selektive Anreize für ökonomische Innovationen, die die Regierungen setzen. Hierzu gehören die staatliche Förderung der Wissenschaft und eine umfassende Bildungspolitik, die gut ausgebildete Arbeitskräfte für die nordischen Arbeitsmärkte zur Verfügung stellt. Aber auch schon weitaus älteren Weichenstellungen sind die ökonomischen Erfolge zu verdanken. Mit der frühen Orientierung der nordischen Ökonomien auf den weitgehend öffentlichen Dienstleistungssektor konnten Entwicklungspotenziale eröffnet werden, von denen die nordischen Ökonomien sowie die nordische Beschäftigung bis auf den heutigen Tag zehren.

\section{Aktive Arbeitsmarktpolitik und Flexicurity}

Aktive Arbeitsmarktpolitik ist vor allem ein schwedisches Erfolgsrezept. Seit den ökonomischen Krisen der 1970er Jahre setzten die schwedischen Regierungen auf die Aktivierung der Erwerbsbevölkerung zur Belebung der Arbeitsmarktdynamik. Diese durchaus kostspielige Politik (vgl. die AAMP-Daten in Tabelle 2) war ein zentraler Pfeiler des sogenannten Rehn-MeidnerModells, mit dem unter den Prämissen ökonomischer Stabilität und geringer Inflation die ökonomische Restrukturierung ohne Massenarbeitslosigkeit erreicht werden sollte (Milner/Wadensjö 2001). Insofern kann kaum davon gesprochen werden, dass aktive Arbeitsmarktpolitik eine neue Erfindung des Nordens sei, deren historische Wurzeln reichen zurück bis in die 1960er Jahre.

Allerdings stehen gegenwärtig bei der nordischen Arbeitsmarktpolitik neue Ziele im Vordergrund. Während früher die Aktivierung vor allem darauf ausgerichtet war, die regionale Mobilität der Erwerbsbevölkerung zu steigern bzw. auf öffentliche Beschäftigungsmaßnahmen zu setzen, sind heutzutage vor allem Aus- und Weiterbildungsmaßnahmen Schwerpunkte einer ar- 
beitsmarktpolitischen Aktivierungs- bzw. „Human-Investment“-Strategie. Zudem werden im Wechselspiel zwischen aktiver und passiver Arbeitmarktpolitik auch im Norden immer stärker Elemente der „Konditionierung" (Clasen/Clegg 2007) eingesetzt. Dies hat zur Folge, dass bürokratischer Druck gegenüber den Arbeitslosen aufgebaut wird, Beschäftigung zu verschlechterten Bedingungen anzunehmen. Am deutlichsten sind dieser Politikmix sowie die Akzentverschiebung der aktiven Arbeitsmarktpolitik am dänischen Beispiel der Flexicurity zu beobachten.

Die dänische Flexicurity knüpft an die schwedische „Arbeitslinie“ in der Arbeitsmarktpolitik an, also an die institutionell abgesicherte Priorität von Beschäftigung bzw. Weiterbildung anstatt passiven Bezuges von Lohnersatzleistung. Die Flexicurity basiert jedoch auf typisch dänischen Besonderheiten der Arbeitsmarktpolitik. Als Flexicurity wird in der Literatur die Verbindung dynamischer Arbeitsmärkte mit bedeutsamer sozialer Absicherung im Falle der Arbeitslosigkeit verbunden. In Dänemark - anders als in den anderen nordischen Ländern - war die staatliche Regulierung der Arbeitsverträge stets sehr gering ausgeprägt. Bereits im historischen „September-Kompromiss" zwischen Arbeitgeberverband und dem Dachverband der dänischen Gewerkschaften aus dem Jahre 1899 wurde das uneingeschränkte Recht der Arbeitgeber zur freien Einstellung und Kündigung von Arbeitskräften seitens der Gewerkschaften akzeptiert. Bis auf den heutigen Tag sticht die geringe Regulierung der dänischen Arbeitsverträge in internationalen Vergleichen hervor (Siegel/ Jochem 2004).

Die dänische Aktivierungspolitik wurde 1993/94 umfassend reformiert (Schwartz 2001; Madsen 2006). Ausgehend von dieser Reform wurde schrittweise die Zeit des passiven Arbeitslosengeldbezuges, d.h. die Zeitspanne ohne Teilnahme an aktivierenden Maßnahmen, auf bis zu ein Jahr (von insgesamt vier Jahren) - bzw. auf bis zu sechs Monate für unzureichend ausgebildete Jugendliche - reduziert. Gleichzeitig wurde die Arbeitsmarktpolitik regionalisiert sowie stärker auf die Bedürfnisse der einzelnen Personen ausgerichtet. Flankiert wird diese Aktivierung jedoch mit einer hohen monetären Absicherung der Arbeitslosen. Nach der einjährigen Phase ohne Pflicht zur Teilnahme an aktivierenden Maßnahmen werden dann allerdings stetig zunehmende Anforderungen an die erwerbslosen Personen gestellt, Arbeitsbzw. Weiterbildungsangebote anzunehmen. Ansonsten droht eine Kürzung des Arbeitslosengeldes. Mit der Definition bestimmter Konditionen gegenüber den erwerbslosen Personen wurde die dänische Arbeitsmarktpolitik verschärft.

Die dänische Flexicurity besitzt auch für die anderen nordischen Länder Vorbildcharakter. Insbesondere in Norwegen und Schweden ist der Arbeitsmarkt vergleichsweise hoch reguliert. Nicht nur die Arbeitgeberverbände, sondern auch Politiker aus dem bürgerlichen sowie zunehmend auch aus dem linken Lager verweisen immer öfter auf das dänische Modell und die dort anzutreffende Arbeitsmarktdynamik. Die geringen Werte für die dänische Langzeitarbeitslosigkeit (vgl. die entsprechenden Daten in Tabelle 1), die auf diese aktive Arbeitsmarktpolitik zurückgeführt werden können, sowie die hohe Zufriedenheit der Dänen mit ihrem System sind Gründe dafür, dass immer mehr nordische Regierungen vom dänischen Vorbild zu lernen bereit sind.

\section{Fazit}

Die beschäftigungspolitische Bilanz des Nordens ist beeindruckend. Dort werden eine umfangreiche soziale Sicherung, weitgehende Lohngleichheit zwischen den Geschlechtern, eine hohe Arbeitsmarktdynamik sowie weitgehende Vollbeschäftigung im Sinne von hoher Beschäftigung und niedriger offener Arbeitslosigkeit gleichzeitig realisiert. Diese gute Performance geht auch auf Weichenstellungen vergangener Zeiten zurück, wozu allemal der frühe und rasche Übergang zu modernen Dienstleistungsökonomien zählt. Bedeutsam ist ferner die prominente Rolle des Staates als Arbeitgeber und Akzentsetzer im Bereich Forschung und Bildung. Neben einem starken öffentlichen Beschäftigungssegment ist die nordische Privatwirtschaft im internationalen Vergleich wettbewerbsfähig - trotz der einzigartig hohen Steuerlast in Skandinavien.

Gleichwohl ist die Steuerpolitik die Achillesferse des nordischen Modells. Mit zunehmendem Steuerdruck und Steuerwettbewerb in Europa sowie dem Erstarken der Parteien, die sich für rapide Steuer- senkungen einsetzen, wird die Finanzierung des nordischen Modells prekär. Dies hätte Auswirkungen auf die öffentliche Beschäftigung, die Finanzierung der Arbeitsmarktpolitik sowie die politisch intendierte steuerpolitische Umverteilung zum Zwecke der Armutsbekämpfung und Sicherung der Einkommensgleichheit. Gleichwohl sind die gegenwärtigen institutionellen und politischen Absicherungen des nordischen Modells (noch) intakt. Auch bürgerliche Regierungen haben bislang keine weitreichende Neujustierung dieser Grundlagen erwirken können bzw. wollen. Im Norden scheint die kulturell fundierte (protestantische) Arbeitsethik noch so stark verankert und in den Präferenzen der Bürger sowie Politiker unterschiedlicher Parteienzugehörigkeit festgeschrieben zu sein, dass eine Zäsur der solidarischen Arbeitsgesellschaft (noch) nicht auf der politischen Agenda steht. Dies ist ein Indiz dafür, dass auch im Zeitalter der sogenannten Globalisierung bzw. der ökonomischen Entgrenzung Politikwege umsetzbar sind, die Ziele der gemeinschaftlichen Solidarität verfolgen. Allerdings steht und fällt eine solche Politik mit der Bereitschaft der Bürger, die hohe Steuerbelastung zu schultern.

Die Leistungskraft des nordischen Modells ist beeindruckend. Sollte die bundesdeutsche Politik also vom Norden lernen, wenn doch auch die angelsächsischen Länder und die Schweiz (als lange vernachlässigtes Beschäftigungswunder in Europa) eine beeindruckende Arbeitsmarktperformance aufweisen können? Letztere - und das sollte betont werden - erkaufen ihre Erfolge allerdings durch Einkommensungleichheit, soziale Verwerfungen, prekäre Arbeitsverhältnisse und weit verbreitete Armut. Gleichwohl scheint sich die bundesdeutsche Politik jüngst eher dem angelsächsischen Modell angenähert zu haben, zumindest wenn zentrale Aspekte der Arbeitsmarktpolitik vergleichend analysiert werden (Dingeldey 2007). In Deutschland fehlt es neben politischen Machtkonstellationen (die Arbeiterbewegung hierzulande ist relativ schwach) und programmatischen Eigenheiten der Regierungsakteure an zentralen politischen und kulturellen Bedingungen für einen nordischen Entwicklungsweg: Zum einen mangelt es an der Akzeptanz einer umverteilenden und hoch belastenden Steuerpolitik. Zum zweiten fehlen sowohl die institutionellen Grundlagen als auch die Unterstützung seitens der Bevölkerung für einen aktiven (und mög- 
lichst kreativen) Staat. Drittens liegt die große Aufgabe eines Übergangs des deutschen Modells mit dem jetzt noch dominierenden Sektor der verarbeitenden Industrie hin zu einer offenen und dynamischen Dienstleistungsökonomie noch vor uns.

Damit kann die These formuliert werden, dass sicherlich vom Norden zu lernen wäre (wenn die politischen Akteure dies wollten), allerdings scheinen die politi- schen und institutionellen Barrieren für eine erfolgreiche Adaption des nordischen Modells in Deutschland sehr hoch zu sein. Eine Politik des "Förderns und Forderns" ist in Deutschland auf halbem Wege stecken geblieben. Die Aktivierung des Arbeitsmarktes mit der tendenziellen Senkung der Langzeitarbeitslosigkeit ist im Norden nicht zum budgetären Nulltarif zu haben, sondern erfordert nicht unerheb- liche monetäre Akzentsetzungen im Bereich der aktiven Arbeitsmarktpolitik sowie in den Bereichen Bildung und Forschung. Insofern mischt sich die in diesem Beitrag zum Ausdruck gebrachte Bewunderung des nordischen Modells mit einer Skepsis hinsichtlich möglicher Lernerfolge in Deutschland.

\section{LITERATUR}

Becker, U. (2008): Was ist dran am skandinavischen Modell? Eine vergleichende Betrachtung, in: Leviathan 36, S. 229-248

Becker, U./Schwartz, H. (Hrsg.) (2005): Employment Miracles. A Critical Comparison of the Dutch, Scandinavian, Swiss, Australian and Irish Case versus Germany and the US, Amsterdam

Clasen, J./Clegg D. (2007): Levels and Levers of Conditionality: Measuring Change within Welfare States, in: Clasen, J./Siegel, N. A. (Hrsg.): Investigation Welfare State Change. The 'Dependent Variable Problem' in: Comparative Analysis, Cheltenham, S. 166-197

Dingeldey, I. (2007): Between Workfare and Enablement - The different paths to Transformation of the Welfare State: A Comparative Analysis of Activating Labour Market Policies, in: European Journal of Political Research 46, S. 823-851

Elvander, N. (2002): The Labour Market Regimes in the Nordic Countries: A Comparative Analysis, in: Scandinavian Political Studies 25, S. 117-137 Ganghof, S. (2006): The Politics of Income Taxation, Colchester Ganghof, S. (2007): The Political Economy of High Income Taxation. Capital Taxation, Path Dependence, and Political Institutions in Denmark, in: Comparative Political Studies 40, S. 1059-1084

Green-Pedersen, C. (2002): New Public Management Reforms of the Danish and Swedish Welfare States: The Role of Different Social Democratic Responses, in: Governance 15, S. 271-294

Hassel, A. (2006): Wage Setting, Social Pacts and the Euro. A New Role for the State, Amsterdam

Iversen, T./Wren, A. (1998): Equality, Employment, and Budgetary Restraint: The Trilemma of the Service Economy, in: World Politics 50, S. $507-546$

Jochem, S. (1998): Die skandinavischen Wege in die Arbeitslosigkeit. Kontinuität und Wandel der nordischen Beschäftigungspolitik im internationalen Vergleich, Opladen

Jochem, S. (2003): Konzertierung und Parteienwettbewerb: Das schwedische Modell im Wandel, in: Jochem, S./Siegel, N. A. (Hrsg.): Konzertierung, Verhandlungsdemokratie und Reformpolitik im Wohlfahrtsstaat. Das Modell Deutschland im Vergleich, Opladen, S. 271-310

Jochem, S. (2006): Die Reichstagswahl 2006 als Zäsur der schwedischen Parteiengeschichte, in: Nordeuropaforum 2, S. 5-24

Jochem, S. (i. E.): Soziale Pakte in Europa, in: Willems, U./von Winter, T./ Rehder, B. (Hrsg.): Interessenvermittlung in Politikfeldern im Wandel. Befunde aus Verbände- und Policyforschung zur Bundesrepublik Deutschland und in vergleichender Sicht, Wiesbaden
Kenworthy, L. (2000): Quantitative Indicators of Corporatism: A Survey and Assesment, MPIfG Discussion Paper 00/4, Köln

Klitgaard, M. B. (2007): Why Are They Doing It? Social Democracy and Market-Oriented Welfare State Reforms, in: West European Politics 30, S. 172-194

Madsen, P. K. (2006): How Can It Possibly Fly? The Paradox of a Dynamic Labour Market in a Scandinavian Welfare State, in: Campbell, J. L./ Hall, J. A./Pedersen, O. K. (Hrsg.): National Identity and the Varieties of Capitalism. The Danish Experience, Montreal, S. 321-355

Merkel, W./Egle, C./Henkes, C./Ostheim, T./Petring, A. (2006): Die Reformfähigkeit der Sozialdemokratie. Herausforderungen und Bilanz der Regierungspolitik in Westeuropa, Wiesbaden

Milner, H./Wadensjö, E. (Hrsg.) (2001): Gösta Rehn and the Swedish Model at Home and Abroad, Aldershot

OECD (2008a): Employment Outlook, Paris

OECD (2008b): Employment in Government in the Perspective of the Production Costs of Goods and Services in the Public Domain, GOV/PGC/PEM1, Paris

OECD (2008c): Factbook, Paris, download http://oberon.sourceoecd. org/vl=1192102/cl=17/nw=1/rpsv/factbook/ index.htm>

Palme, J. (1999): The Nordic Model and the Modernisation of Social Protection in Europe, Copenhagen

Schwartz, H. M. (2001): The Danish „Miracle“. Luck, Pluck, or Stuck? in: Comparative Political Studies 34, S. 131-155

Siaroff, A. (1999): Corporatism in 24 industrial democracies: Meaning and Measurement, in: European Journal of Political Research 36, S. 175205

Siegel, N. A. (2005): Social Pacts Revisited: "Competitive Concertation" and Complex Causality in Negotiated Welfare State Reforms, in: European Journal of Industrial Relations 1, S. 107-126

Siegel, N. A./Jochem, S. (2004): Staat und Markt im internationalen Vergleich, in: Zintl, R./Czada, R. (Hrsg.): Politik und Markt, PVS Sonderheft 2003, Wiesbaden, S. 351-388

Sipilä, J. (Hrsg.) (1997): Social Care Services: The Key to the Scandinavian Welfare Model, Aldershot

Visser, J./Hemerijck, A. C. (1998): Ein holländisches Wunder? Reform des Sozialstaates und Beschäftigungswachstum in den Niederlanden, Frankfurt/M./New York 